\title{
The pre-supernova evolution of rotating massive stars
}

\author{
Alexander Heger ${ }^{1}$, Stan E. Woosley ${ }^{2}$, and Norbert Langer ${ }^{3}$ \\ ${ }^{1}$ Department of Astronomy and Astrophysics, Enrico Fermi Institute, \\ The University of Chicago, 5640 S. Ellis Ave, Chicago, IL 60637, USA \\ ${ }^{2}$ Astronomy and Astrophysics Department, University of California, \\ Santa Cruz, CA 95064, USA \\ ${ }^{3}$ Sterrekundig Instituut, Universiteit Utrecht, \\ Princetonplein 5, NL-3584 CC Utrecht, Nederland
}

\begin{abstract}
Massive stars are born rotating rigidly with a significant fraction of critical rotation at the surface. Consequently, rotationally-induced circulation and instabilities lead to chemical mixing in regions that would otherwise be stable, as well as a redistribution of angular momentum. Differential rotation also winds up magnetic fields, causing instabilities that can power a dynamo and magnetic stresses that lead to additional angular momentum transport. We follow the evolution of typical massive stars, their structure and angular momentum distribution, from the zero-age main sequence until iron core collapse. Without the action of magnetic fields, the resulting angular momentum is sufficiently large to significantly affect the explosion mechanism and neutron star formation. Sub-millisecond pulsars result that could encounter the $r$-mode instability. In helium cores massive enough, at least at low metalicity, the angular momentum is also sufficiently great to form a centrifugally supported accretion disk around a central black hole, powering the engine of the 'collapsar' model for GRBs. Including current estimates of the effect of magnetic fields still allows the formation of rapidly rotating $(\sim 5-10 \mathrm{~ms})$ pulsars, but might leave too little angular momentum for collapsars.
\end{abstract}

\section{Introduction}

Massive stars, those that are born with initial masses of more than about $8 \mathrm{M}_{\odot}$, are known to rotate on the main sequence at a significant fraction of their breakup velocity. Typical rotation velocities are of the order of $200 \mathrm{~km} \mathrm{~s}^{-1}$ (e.g., Fukuda 1982), around $30 \%$ of critical rotation. Once the star is born, its center generally evolves to increasing central density and temperature. This overall contraction is interrupted by phases of nuclear fusion - hydrogen to helium, helium to carbon and oxygen, then carbon, neon, oxygen and silicon burning, until finally iron is produced and the core collapses. Each fuel burns first in the center, then in a shell. The time scale for helium burning is about ten times shorter than that of hydrogen burning, mostly because of the lower energy release per unit mass. However, the time scale of the burning stages beyond central helium-burning is radically reduced by thermal neutrino losses that carry 
away energy in situ, instead of requiring that it be transported to the stellar surface. These losses increase with temperature, roughly $\propto T^{9}$ (see Woosley, Heger \& Weaver 2002 for a more extended review). When the star has built up a large enough iron core, exceeding its Chandrasekhar mass, it collapses to form a neutron star or a black hole. A supernova explosion may result (Fryer, these Proceedings), or even, in rare cases, a gamma-ray burst.

Two key uncertainties in this generally accepted picture of massive stellar evolution are just how rotation affects the evolution and what the distribution of angular momentum is in the interior when the star dies. The first affects the structure and composition of the pre-supernova star and understanding the latter is essential in order to predict the rotation rates of young pulsars and to determine which stars might produce gamma-ray bursts.

In this article we review recent efforts to model the evolution of rotating massive stars. We also present some recent results that include magnetic fields torques and discuiss their implication for pulsar rotation and gamma-ray burst engines ('collapsars').

\section{Numerical method}

For this purpose, we use a 1-D implicit hydrodynamic stellar evolution code (KEPLER; Weaver, Zimmerman \& Woosley 1978). To this code we add angular momentum as a local variable and assume 'shellular' rotation (Endal \& Sofia 1978; Chaboyer \& Zahn 1992; Zahn 1992; Meynet \& Maeder 1997). Mixing and angular momentum transport are solved in diffusion approximation. We account, in an approximate way, for convection and semiconvection, dynamical and secular shear instabilities, the Goldreich-Schubert-Fricke instability, EddingtonSweet circulation and Solberg-Høiland instability (Endal \& Sofia 1978; Pinsonneault et al. 1989; Heger, Langer \& Woosley 2000). The uncertain efficiencies of rotationally-induced mixing processes and of the inhibiting effects of composition gradients have been gauged to reproduce the average helium and nitrogen enrichments of main-sequence O- and B-type stars (Heger et al. 2000).

To study the effect of magnetic fields, we have now implemented the formalism of Spruit (2002). In contrast to earlier work by Spruit \& Phinney (1998), this prescription includes the stabilizing effect of composition and temperature stratification and allows a much weaker radial field than the toroidal field. A dynamo process is assumed to act on a time scale short compared to that of the stellar evolution, so that the magnetic field, and the resulting stress and mixing, are only a function of the current local conditions (stratification, rotation velocity, and shear).

\section{Pre-supernova evolution}

\subsection{Hydrogen burning}

Rotation leads to a reduction of effective gravity in the star and therefore they start with lower luminosity and surface temperature than their non-rotating counterparts. Also the lifetime early during hydrogen burning is extended (e.g., Endal \& Sofia 1976; Heger et al. 2000; see Maeder \& Meynet 2000 for a review 
Table 1. Evolution of angular momentum $(j)$, radius $(R)$, angular velocity $(\omega)$ and its ratio to Keplerian angular velocity $\left(\omega / \omega_{\text {Kepler }}\right)$ at a mass coordinate of $M=1.7 \mathrm{M}_{\odot}$, and central density in a $20 \mathrm{M}_{\odot}$ star. For the neutron star we assume a radius of $12 \mathrm{~km}$ and a moment of inertia of $I=0.32 M R^{2}$.

\begin{tabular}{lrcrrl}
\hline \hline $\begin{array}{l}\text { evolutionary } \\
\text { state }\end{array}$ & $\begin{array}{c}j \\
\left(\mathrm{~cm}^{2} \mathrm{~s}^{-1}\right)\end{array}$ & $\begin{array}{c}R \\
(\mathrm{~cm})\end{array}$ & $\begin{array}{r}\rho_{\text {central }} \\
\left(\mathrm{g} \mathrm{cm}^{-3}\right)\end{array}$ & $\begin{array}{r}\omega \\
\left(\mathrm{rad} \mathrm{s}^{-1}\right)\end{array}$ & $\omega / \omega_{\text {Kepler }}$ \\
\hline ZAMS & $1.1 \times 10^{17}$ & $5.8 \times 10^{10}$ & 4.8 & $5 \times 10^{-6}$ & 0.046 \\
H-depletion & $2 \times 10^{16}$ & $4.3 \times 10^{10}$ & 12.5 & $1.6 \times 10^{-5}$ & 0.01 \\
He-depletion & $1.3 \times 10^{16}$ & $7.2 \times 10^{9}$ & 3000 & $3.8 \times 10^{-4}$ & 0.015 \\
pre-supernova & $1.2 \times 10^{16}$ & $2.2 \times 10^{8}$ & $3.9 \times 10^{9}$ & 0.37 & 0.08 \\
neutron star & $\left(10^{16}\right)$ & $1.2 \times 10^{6}$ & $4 \times 10^{14}$ & $1 \times 10^{4}$ & 0.9 \\
\hline \hline
\end{tabular}

of stellar rotation). However, later on, rotationally induced mixing enriches the envelope in helium: the resulting higher mean molecular weight increases the luminosity of the star (Kippenhahn \& Weigert 1990), so that at the terminal age main sequence the star can become more luminous (Heger \& Langer 2001).

Besides mixing of nitrogen and helium, which we have used to gauge the mixing processes, also other species show the effect of the rotationally induced mixing and can be used as a diagnostic tool. We see surface depletion of carbon and oxygen, going along with the nitrogen enrichment, and some enrichment in sodium, also being made in the center of the star (Heger \& Langer 2001).

A valuable diagnostic tool is the depletion of light elements: lithium, boron, and beryllium (see also Fliegner, Langer \& Venn 1996; Pinsonneault 1997). They are already destroyed at a few million $\mathrm{K}$, i.e., not too deep in the stellar envelope. In rotating stars, the mixing brings depleted material from deep inside the star to the surface, reducing the abundance of these elements. Interestingly, in nonrotating stars one expects that these fragile elements entirely disappear when the outer layer of the star that were never exposed to high enough temperature to destroy them are eroded away by stellar winds, typically for stars above $\sim 20 \mathrm{M}_{\odot}$. However, in rotating stars some of the light elements can actually be preserved in the outer layers, though at rather low abundance. This is because, as the outer stellar layers are being carried away by the stellar wind, layers below get closer to the surface and cool down. Light elements now being mixed into these regions can be preserved there (Heger \& Langer 2001).

We find that a strong gradient in mean molecular weight forms close to the maximum extent of the convective hydrogen core (i.e., at the ZAMS) acting as a barrier for transport of angular moment and for mixing. At the end of central hydrogen burning we find, e.g., in a $15 \mathrm{M}_{\odot}$ star, a core that spins about three times faster than the envelope. The slow-down of the envelope is in part due to angular momentum loss by winds, in part due to expansion of the envelope, i.e., an increase of its moment of inertia.

\subsection{Helium burning}

In helium burning an important effect of rotation is that it may suppress the formation of separate convective layers in the helium core that do not mix because of different mean molecular weight. The outer edge of the helium core is 


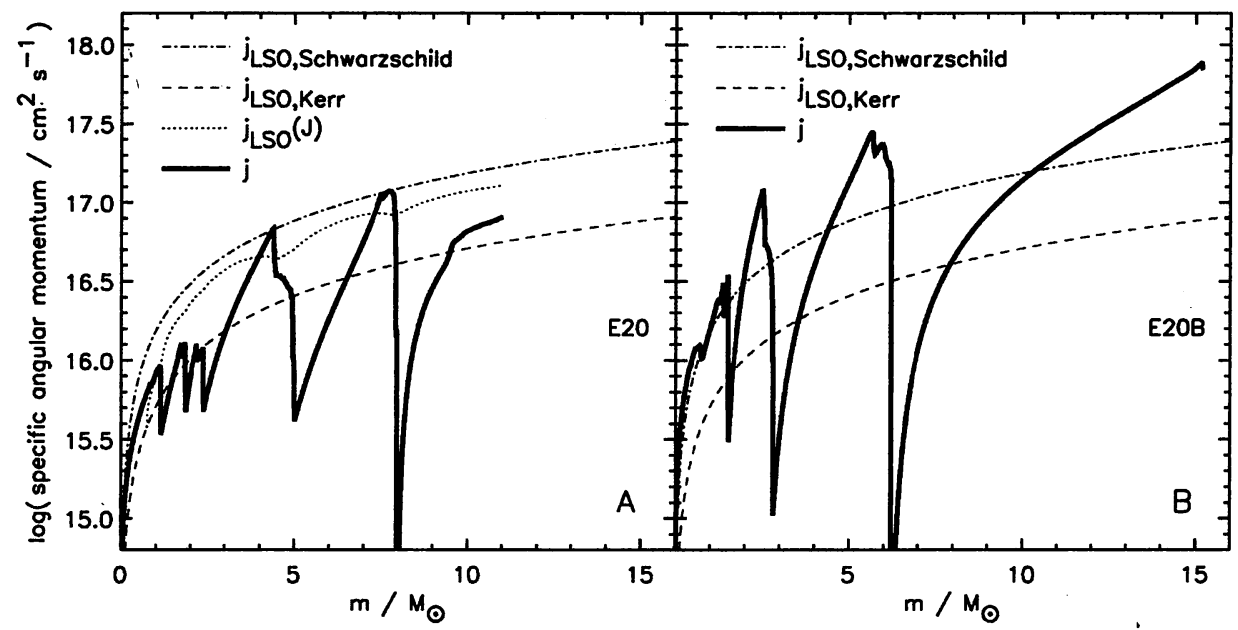

Figure 1. Distribution of equatorial specific angular momentum at onset of core collapse in a $20 \mathrm{M}_{\odot}$ star. We shows two cases: with (Panel B) and without (Panel $A$ ) inhibition of rotationally induced mixing processes by composition barriers. As a measure for the magnitude of rotation we give the specific angular momentum of the last stable orbit around a (non-rotating) Schwarzschild black hole (dashed curve), and for a (maximum rotating) Kerr black hole (dash-dotted curve). The dotted curve gives the last stable orbit of a black hole, that has a spin parameter corresponding to the mass and angular momentum below each mass coordinate.

stabilized against rotationally induced mixing by a strong entropy gradient at the hydrogen shell. Some rotationally induced mixing of protons downward occurs, but the protons rapidly burn due to the higher temperatures and therefore do not penetrate deep.

Accordingly, we also do not find much loss of angular momentum from the core, though we see some redistribution of angular momentum within the helium core as it contracts. However, the average angular momentum of the core decreases as it grows by hydrogen burning and incorporates outer layers deficient in angular momentum (Heger 1998).

\subsection{Late burning stages}

In the late burning stages, carbon, neon, oxygen and silicon burning, the time scale of burning accelerates compared to the earlier stages, because energy losses by neutrinos become important. For the amount of angular momentum in our models, the evolution time scale becomes so short that rotationally induced mixing processes are unimportant. Similarly, convection is the only process that keeps rapid enough for angular momentum redistribution. Therefore, at the time of core collapse, we find a rotation profile that reflects the location and extent of the last burning shell in each region of the star. The resulting rotation profile shows regions of enhanced angular momentum at the outer edged of (former) convective shells and low angular momentum at their lower bounds (Figure 1; Heger et al. 2000). 
Table 2. Rotation rates of observed young pulsars. Periods are taken from Marshall et al. (1998). We assume rigid rotation, radius $R=12 \mathrm{~km}$, and moment of inertia $I=0.32 M R^{2}$, and $J=2 \pi I / P, j=J / M, M \approx 1.4 \mathrm{M}_{\odot}$ (gravitational mass).

\begin{tabular}{lrcc}
\hline \hline pulsar state & $\begin{array}{c}P \\
(\mathrm{~ms})\end{array}$ & $\begin{array}{c}j \\
\left(\mathrm{~cm}^{2} \mathrm{~s}^{-1}\right)\end{array}$ & $\begin{array}{c}J \\
(\mathrm{ergs})\end{array}$ \\
\hline PSR J 0537-69 (N 157B, LMC) & 16 & $1.8 \times 10^{14}$ & $5.2 \times 10^{47}$ \\
PSR B 0531+21 (Crab) & 33 & $8.8 \times 10^{13}$ & $2.5 \times 10^{47}$ \\
PSR B 0540-69 (LMC) & 50 & $5.8 \times 10^{13}$ & $1.7 \times 10^{47}$ \\
PSR B 1509-58 & 150 & $1.9 \times 10^{13}$ & $5.5 \times 10^{46}$ \\
\hline \hline
\end{tabular}

\section{Supernovae and remnants}

The angular momentum found in these stars can influence the collapse and explosion of supernovae. For a $15 \mathrm{M}_{\odot}$ star, Fryer \& Heger (2000) found that the bounce following collapse was weakened, and a less energetic explosion resulted. For more details see Fryer (these Proceedings).

In Table 2 we give a list of rotation rates of young pulsars and their estimated angular momentum. Compared to these, our stars have excessive angular momentum (Figure 1), therefore some spin-down mechanism is required to explain the observed young pulsar rotations. A solution to this discrepancy might be that the $r$-mode instability (e.g., Lindblom, Owen \& Morsink 1998; Lindblom, Tohline \& Vallisneri 2001) could carry away the angular momentum by gravitational radiation within about the first year after collapse when the neutron star is still hidden inside the supernova explosion.

A second possibility is that we underestimate the angular momentum transport inside the stars, i.e., in particular the angular momentum loss from the core. This could be due to hydrodynamical instabilities we have neglected, or due to the action of magnetic fields.

\section{Maǵnetic fields}

The pioneering work by Spruit \& Phinney (1998) assumed that an initial seed field was wound up into a toroidal field and that magnetic instabilities led to a radial field of comparable strength. They estimated that the star remained essentially in rigid rotation until central carbon burning. Only then did the inner regions decouple and preserve local angular momentum. Their treatment of magnetic fields resulted in estimated pulsar rotation rates of $\sim 100 \mathrm{~s}-$ much too slow. If additionally braking due to mass loss by stellar winds had been considered, the resulting neutron star rotation would have been even less.

We performed the first calculations that employ the improved treatment of magnetic fields by Spruit (2002; see §2.). An upper limit on the resulting neutron star rotation rates is given in Table 3 , assuming that all angular momentum present in the core at time of collapse is engulfed into the neutron star. However, some angular momentum could be lost during neutron star formation, and some 
Table 3. Rotation periods of neutron stars from stars evolved taking into account magnetic fields. We assume rigid rotation, that the inner $1.6 \mathrm{M}_{\odot}$ of the star collapse to a neutron star of $1.4 \mathrm{M}_{\odot}$ gravitational mass and a radius of $R=12 \mathrm{~km}$ and a moment of inertia of $I=0.32 M R^{2}$. We give the resulting pulsar period $(P)$, average specific angular momentum $(j=J / M)$ and total angular momentum $(J)$.

\begin{tabular}{cccc}
\hline \hline $\begin{array}{c}\text { initial mass } \\
\left(\mathrm{M}_{\odot}\right)\end{array}$ & $\begin{array}{c}P \\
(\mathrm{~ms})\end{array}$ & $\begin{array}{c}j \\
\left(\mathrm{~cm}^{2} \mathrm{~s}^{-1}\right)\end{array}$ & $\begin{array}{c}J \\
(\mathrm{ergs})\end{array}$ \\
\hline 15 & 7.7 & $3.9 \times 10^{14}$ & $1.1 \times 10^{48}$ \\
20 & 5.5 & $5.5 \times 10^{13}$ & $1.5 \times 10^{48}$ \\
25 & 4.0 & $7.5 \times 10^{13}$ & $2.1 \times 10^{48}$ \\
\hline \hline
\end{tabular}

$\sim 20 \%$ additionally is carried away by neutrinos. Considering that the initial rotation period of young pulsars may have been as low as $10 \mathrm{~ms}$, the amount of angular momentum in these magnetic pre-supernova models seems plausible. However, we caution that effect of the magnetic fields is still very uncertain.

\section{Collapsars}

With increasing mass of the core more energy is required to explode the star. Eventually, the mechanism that powers the explosion of the lighter cores may fail (e.g. ., Fryer \& Kalogera 2001) and a black hole may form directly, or within a few to a few $10 \mathrm{~s}$ by material falling back onto the neutron star. If the material that falls toward the black hole has sufficient angular momentum it cannot directly fall into the black hole, but rather forms an accretion disk, which might become a powerful engine for bipolar energy outflow perpendicular to the disk ('collapsar', Woosley 1993). This may result in a gamma-ray burst (GRB) if the star has no hydrogen envelope (MacFadyen \& Woosley 1999) or a jet-powered energetic supernova (MacFadyen, Woosley \& Heger 2001).

The specific angular momentum required to produce such accretion disks, however, is much larger than what would be needed to produce pulsars with initial rotation rates of $\sim 10 \mathrm{~ms}$. So, if magnetic fields are as efficient in transporting angular momentum as assumed by Spruit (2002), it will be difficult to produce collapsars for the same progenitor scenario that makes a normal neutron star. Moreover, test calculations indicate that for bare helium stars, the likely progenitors of GRBs, their high mass loss combined with magnetic fields leads to excess slow-down for collapsars. Accretion of material in a late Case A (end of central hydrogen burning) binary system may significantly speed up the core of the secondary star (see also Joss \& Becker, 2003, for a discussion of core spin-up by accretion and gravity waves), but critical point will remain whether magnetic fields stay efficient beyond central carbon burning and brake the core rotation too much for collapsars. 


\section{Summary}

During central hydrogen burning rotation leads to mixing processes that result in observable surface 'anomalies' relative to non-rotating 'pristine' interstellar material. At the end of central hydrogen burning, the core of non-magnetic stars typically rotates a few times faster than the envelope. During helium burning, the core is not slowed down much, and after central helium burning, only rotation is fast enough to mix and to transport angular momentum. Too rapidly rotating young neutron stars could result, but enough angular momentum may be available for collapsars, at least for metal-poor stars with low mass loss.

When magnetic fields according to Spruit (2002) are taken into account, the picture reverses: the predicted angular momentum is compatible with some fast young pulsars (Table 2), but too little for collapsars. It is promising, however, that the bigger stars seem to result in faster rotating cores (Table 3 ) and it is the big stars that are assumed to become collapsars and possibly power GRBs while the smaller stars should leave neutrons stars and pulsars.

Acknowledgments. We thank Henk Spruit for many fruitful discussions and help implementing his description of magnetic fields in the stellar interiors. This work is partially supported by the Department of Energy under grant B341495 to the Center for Astrophysical Thermonuclear Flashes at the University of Chicago, by the NSF (AST 02-06111), and the DOE ASCI Program (B347885). AH acknowledges supported by a Fermi Fellowship of the Enrico Fermi Institute at The University of Chicago.

\section{References}

Chaboyer, B., Zahn, J.-P. 1992, A\&A 253, 173

Endal, A.S., Sofia, S. 1976, ApJ 210, 184

Endal, A.S., Sofia, S. 1978, ApJ 220, 279

Fliegner, J., Langer, N., Venn, K.A. 1996, A\&A 308, 13

Fryer, C.L., Heger, A. 2000, ApJ 541, 1033

Fryer, C.L., Kalogera, V. 2001, ApJ 554, 548

Fukuda, I. 1982, PASP 94, 271

Heger, A. 1998, PhD thesis, Technische Universität München, BRD

Heger, A., Langer, N. 2001, ApJ 544, 1016

Heger, A., Langer, N., Woosley, S.E. 2000, ApJ 528, 368

Joss, P.C., Becker, J.A. 2003, in: W. Hillebrandt \& B. Leibundgut (eds.), From Twilight to Highlight, ESO Astrophysics Symposia (Berlin: Springer), in press

Kippenhahn, R., Weigert, A. 1990, Stellar Structure and Evolution (Berlin: Springer).

Lindblom, L., Owen, B.J., Morsink, S.M. 1998, Phys. Rev. Letters 80, 4843

Lindblom, L., Tohline, J.E., Vallisneri, M. 2001, Phys. Rev. Letters 86, 1152

MacFadyen, A.I., Woosley, S.E. 1999, ApJ 524, 262

MacFadyen, A.I., Woosley, S.E., Heger, A. 2001, ApJ 550, 410

Maeder, A., Meynet, G., 2000, Ann. Review Astron. Astrophys. 38, 143

Marshall, F.E., Gotthelf, E.V., Zhang, W., Middleditch, J., Wang, Q.D. 1998, ApJ (Letters) 499, L179

Meynet, G., Maeder, A. 1997, A\&A 321, 465 
Pinsonneault, M.H. 1997, Ann. Review Astron. Astrophys. 35, 557

Pinsonneault, M.H., Kawaler, S.D., Sofia, S., Demarque, P. 1989, ApJ 338, 424

Spruit, H.C. 2002, A\&A 381, 923

Spruit, H.C., Phinney, E.S. 1998, Nature 393, 139

Weaver, T.A., Zimmerman, G.B., Woosley, S.E. 1978, ApJ 225, 1021

Woosley, S.E. 1993, ApJ 405, 273

Woosley, S.E., Heger, A., Weaver, T.A. 2002, Rev. Mod. Phys. 74, 1015

Zahn, J.-P. 1992, A\&A 265, 115

\section{Discussion}

MAEDER: In the advanced stages, you have enormous differences of angular velocity between the core and the envelope. We also get this kind of results, but I always have some doubts about that to be stable.

HEGER: This apparent steep gradient in the rotation profile is an artifact of showing angular velocity as a function of mass coordinate in the star. This steep drop in rotation (10 orders of magnitude) corresponds to a similar drop in density. This means that over this drop, the radius actually changes by several orders of magnitude (at to the base of the convective envelope). Therefore, the rotation has only a rather shallow gradient with respect to radius, and therefore no dynamical instabilities occur. The stratification is stabilization by a sub-adiabatic temperature gradient.

SONNEBORN: Does the asymmetry in the exploding core of the rotating star after corecollapse persist to the surface layer ejecta?

FRYER: The explosion will spherize later on, but the Ni ejecta may show/keep asymmetry.

LANGER: Comment: your non-magnetic models show lower specific angular momenta in the core for higher initial mass. It seems to me that, if you would consider initial masses of interest for the collapse models, you would not find enough angular momentum for it to work. Question: Your magnetic models show the reverse trend: higher angular momenta for higher mass. Is this trend easily understood?

HEGER: As to your question: this is likely due to $(i)$ the shorter evolution time-scales in the late stages of more massive stars, and (ii) lower density and shallower density gradient $\rightarrow$ shallower gradient of rotation profile $\rightarrow$ less angular momentum transport. Note that in these stars the angular momentum transport due to dynamo action remains important at least through carbon burning. The trend seen in the 'non-magnetic stars' is dominated by the increasing mass loss in more massive star, while in their late stages angular momentum transport outside convective region is slow during central helium burning and essentially stalls after central helium depletion. 